Revue d'histoire de l'Amérique française

Q4. REVUE D.HISTOIRE DE L'AMÉRIQUE FRANÇAISE

\title{
Crise du logement et action catholique à Montréal, 1940-1960
}

\section{Jean-Pierre Collin}

Volume 41, numéro 2, automne 1987

URI : https://id.erudit.org/iderudit/304550ar

DOI : https://doi.org/10.7202/304550ar

Aller au sommaire du numéro

\section{Éditeur(s)}

Institut d'histoire de l'Amérique française

ISSN

0035-2357 (imprimé)

1492-1383 (numérique)

Découvrir la revue

Citer cet article

Collin, J.-P. (1987). Crise du logement et action catholique à Montréal, 1940-1960. Revue d'histoire de l'Amérique française, 41(2), 179-203.

https://doi.org/10.7202/304550ar

\section{Résumé de l'article}

Durant les années quarante et cinquante, le logement est un des principaux champs d'action et de luttes pour les organisations canadiennes-françaises d'action sociale catholique, surtout pour la Ligue ouvrière catholique. Inspirés à la fois par les discours des hygiénistes réformistes du début du siècle, par les thèses de certains urbanistes et architectes et par la morale familiale catholique, les animateurs de l'action catholique proposent et veulent imposer la propriété de l'unifamiliale détachée comme modèle de vie résidentielle. « A chaque famille, sa maison ", tel est le slogan le plus fréquemment utilisé. Cet article examine les lignes de force du projet d'action catholique - ses revendications, ses actions et son discours. On y trace, plus particulièrement, un portrait des réalisations et des modes successifs de fonctionnement des coopératives d'habitation dans la région métropolitaine de Montréal. En conclusion, l'originalité et la spécificité de l'action catholique sont discutées en fonction des traits généraux de la production résidentielle à l'époque.
Tous droits réservés @ Institut d'histoire de l'Amérique française, 1987
Ce document est protégé par la loi sur le droit d'auteur. L'utilisation des services d’Érudit (y compris la reproduction) est assujettie à sa politique d'utilisation que vous pouvez consulter en ligne.

https://apropos.erudit.org/fr/usagers/politique-dutilisation/ 


\title{
CRISE DU LOGEMENT ET ACTION CATHOLIQUE A MONTRÉAL, 1940-1960
}

\author{
JEAN-PIERRE COLLIN \\ INRS-Urbanisation
}

\begin{abstract}
RÉSUMÉ
Durant les années quarante et cinquante, le logement est un des principaux champs d'action et de luttes pour les organisations canadiennes-françaises d'action sociale catholique, surtout pour la Ligue ouvrière catholique. Inspirés à la fois par les discours des hygiénistes réformistes du début du siècle, par les thèses de certains urbanistes et architectes et par la morale familiale catholique, les animateurs de l'action catholique proposent et veulent imposer la propriété de l'unifamiliale détachée comme modèle de vie résidentielle. «A chaque famille, sa maison», tel est le slogan le plus fréquemment utilisé. Cet article examine les lignes de force du projet d'action catholique ses revendications, ses actions et son discours. On y trace, plus particulièrement, un portrait des réalisations et des modes successifs de fonctionnement des coopératives d'habitation dans la région métropolitaine de Montréal. En conclusion, l'originalité et la spécificité de l'action catholique sont discutées en fonction des traits généraux de la production résidentielle à l'époque.
\end{abstract}

\begin{abstract}
In the fourties and the fifties, housing is a major field of action and struggles for frenchcanadian organizations involved in catholic social action, especially in the case of the Ligue ouvrière catholique. Influenced simultaneously by the arguments of hygienist reformers of early 20th century, on one hand, of some contemporary urban planners and architects, on the other hand, and by the catholic familial ethic, group leaders in catholic social action put forward and try to enjoin home ownership of single-family detached house as a model of living conditions. "A home for every family" was the main slogan of the movement. This paper examines the trends of the catholic action project - claims, activities, proposals. Particularly, the realizations and the successive institutional arrangements of housing co-ops in the Montreal metropolitain area are outlined. In conclusion, the distinctive character and the peculiarity of the catholic action are examined in reference to the general features of the housing market at the time.
\end{abstract}

Dans les années trente et après, les organisations canadiennesfrançaises d'action sociale catholique - notamment la Ligue ouvrière catholique (LOC) - interviennent de diverses manières pour améliorer les conditions de la vie urbaine des ouvriers et des salariés en général. Les problèmes du logement sont au centre des préoccupations de ces partisans de la doctrine sociale catholique, relancée en 1931 par Pie XI dans Quadragesimo Anno. Au cours des années quarante et cinquante, ils font du logement un des principaux champs de leur action et de leurs luttes. Ainsi les groupes d'action catholique animent, pendant plusieurs 
années, un vaste mouvement de revendications en faveur d'un «crédit urbain» (parfois appelé le «crédit ouvrier»), une politique d'aide à l'accession à la propriété qui reprend l'esprit de l'aide aux agriculteurs par le crédit agricole. Parallèlement, ils organisent sur le terrain une solution de rechange à l'État providence et aux abus de l'entreprise privée: les coopératives d'habitation.

Durant la guerre et au cours de la décennie qui a suivi, la région métropolitaine de Montréal a connu une grave crise du logement. Dans cet article, nous examinons les paramètres de l'action sociale catholique en regard de cette situation. Après une brève description des difficultés du marché du logement, nous situerons la réponse de l'action catholique dans le contexte du développement de l'intervention du gouvernement fédéral en matière d'habitation. Les deux sections suivantes proposent une analyse des lignes de forces de l'action catholique chapeautée par le slogan «A chaque famille, sa maison», pour la première, et un bilan des coopératives d'habitation dans la région de Montréal, pour la seconde. En conclusion, nous tentons de dégager l'originalité et la spécificité de l'action catholique en fonction des traits généraux de la production résidentielle à l'époque.

\section{LE MARCHÉ DU LOGEMENT À MONTRÉAL, PENDANT ET APRĖS LA GUERRE}

En décembre 1939, l'Office d'initiative économique de Montréal sonne l'alarme: il manquerait 35000 logements dans la métropole ${ }^{2}$. En 1943, le service d'urbanisme estime que la pénurie s'est aggravée et qu'il faut construire d'urgence 50000 logements $^{3}$. Au cours de ces années, la hausse des loyers est nettement supérieure à celle des salaires. Cette situation pénible est la conséquence de la morosité économique des années trente qui avait pratiquement arrêté la construction résidentielle. L'entrée en guerre n'arrange pas les choses puisqu'au rationnement des matériaux et de la main-d'oeuvre en faveur de l'effort de guerre, ce qui bloque la reprise de la construction, s'ajoute l'arrivée à Montréal de 65000 personnes attirées par les emplois dans les usines d'armement (entre 1940 et 1945).

On assiste à un formidable entassement de plusieurs familles dans un même logement. Les chambreurs se multiplient. Les constructions

1 Un tableau plus détaillé du marché montréalais du logement entre 1940 et 1960 est proposé dans Jean-Pierre Collin, La Cité coopérative canadienne-française (Montréal, Presses de l'Université du Québec, 1986), chapitre premier et dans Jean-Pierre Collin, «L'espace urbain de Montréal après la Deuxième Guerre mondiale: mutations et continuités», à paraître en italien dans Storia Urbana.

\footnotetext{
Office d'initiative économique de Montréal, Évolution du taux de vacance 1929-1939 (Montréal, Ville de Montréal, décembre 1941).

Service d'urbanisme de la Ville de Montréal, Le problème de l' habitation à Montréal (19 juillet 1943, miméo).
} 
les plus diverses et les plus saugrenues servent d'abris à des familles à faible revenu. En outre, dans les quartiers ouvriers les logements se détériorent faute d'entretien adéquat.

La fin de la Seconde Guerre mondiale marque une reprise lente mais continue de la construction domiciliaire. Toutefois, la production de logements reste bien en deçà des retards accumulés que les pressions démographiques exercées depuis 1941 empêchent de combler. Les nouveaux ménages familiaux sont si nombreux qu'ils s'adjugent l'ensemble de la production neuve. Par ailleurs, cette croissance du nombre des familles s'accompagne d'une diminution substantielle de leur taille. L'ère de la famille nombreuse étant révolue, le marché du logement connaît de sérieuses difficultés d'ajustement. D'autant plus que, dès les années quarante, le nombre des ménages non familiaux amorce une irrésistible poussée de croissance.

Il n'est donc pas étonnant de constater que dans les premières années de la décennie 1950, l'agglomération de Montréal, comme l'ensemble du Canada urbain, notons-le, vit encore un relatif surpeuplement de ses logements. Ainsi, $20 \%$ des familles de la région métropolitaine cohabitent avec une autre famille ${ }^{4}$. Certains analystes prétendent même que la pénurie de logements dépasse maintenant les 60000 .

Bref, au début des années cinquante, le marché du logement à Montréal n'arrive pas à combler le retard accumulé depuis 1930 et l'attention des pouvoirs publics est requise. C'est le gouvernement fédéral canadien qui prend résolument le leadership de l'intervention étatique $^{5}$. Cependant, entre 1941 et 1954 , tous les paliers de gouvernements ont adopté des programmes d'intervention en matière de logement. Si de nombreux spécialistes et fonctionnaires proclament l'urgence d'aider la production de logements locatifs à loyer raisonnable, les pouvoirs publics ont opté avec une rare unanimité et une constance exemplaire pour des politiques et des programmes de soutien à l'accession à la propriété .

\section{LES PREMIÈRES RÉPONSES À LA CRISE DU LOGEMENT}

Dans le cadre de l'effort de guerre rendu nécessaire par sa participation à la Seconde Guerre mondiale, le Canada est amené à intervenir dans le domaine du logement, notamment pour loger les ouvriers

\footnotetext{
4 Georges Mathews, Évolution générale du marché du logement de la région de Montréal de 1951 à 1976: données synthétiques sur une réussite méconnue (Montréal, INRS-Urbanisation, Études et documents no 17, 1980).

5 Marc-H. Choko, Jean-Pierre Collin et Annick Germain, «Le logement et les enjeux de la transformation de l'espace urbain: Montréal, 1940-1960», première partie, Urban History Review/ Revue d' histoire urbaine, 15,2 (1986): 127-136.

6 Urban History Review/Revue d'histoire urbaine, «Special Issue on the History of Canadian Housing Policy», 15,1 (1986).
} 
des usines de guerre. Il se trouve ainsi à aller dans le sens des revendications des partisans du «welfare state» qui prêchent pour une intervention de l'État fédéral dans le domaine de l'habitation. Au cours des débats qui se déroulent à l'intérieur de l'appareil gouvernemental, l'argument économique a gain de cause sur l'argument social. La pièce maîtresse de la participation fédérale, dont les grandes lignes étaient déjà contenues dans les lois de 1935 et de 1938, est une politique d'encadrement du marché des hypothèques pour soutenir les bénéfices des prêteurs et stabiliser l'industrie de la construction résidentielle.

L'objectif immédiat est de préparer l'après-guerre car on veut éviter de revivre les troubles sociaux de 1918-1919. A plus long terme, l'objectif est double. D'une part, l'intervention gouvernementale doit favoriser la production en série et l'accroissement de la taille des chantiers (et, conséquernment, des entrepreneurs) afin d'appuyer la modernisation et la stabilisation de l'industrie de la construction ${ }^{7}$. D'autre part, la libéralisation du marché hypothécaire permettra de généraliser le statut de propriétaire chez les ménages de la classe moyenne.

Donc, entre 1938 et 1954, l'effet prédominant des diverses lois nationales sur l'habitation ( $\mathrm{LNH})$ aura été de redéfinir, en collaboration avec le capital financier, le régime hypothécaire par l'allongement de la période d'amortissement des prêts jusqu'à 25 ans, parfois même 30 ans, par la réduction progressive du versement initial - qui n'est plus que de $10 \%$ du coût de la construction en $1954-$, par une réduction du taux d'intérêt, et par l'élargissement du marché des hypothèques à de nouvelles institutions financières dont les placements sont assurés par le fédéral.

Néanmoins, au début des années quarante, l'effort de guerre oblige à des interventions beaucoup plus directes auprès des consommateurs et dans le processus de production des logements. Dans le premier cas, malgré les résistances locales (à Montréal en particulier), Ottawa entreprend de contrôler le coût des loyers et de limiter considérablement les droits d'éviction et de reprise de possession par le propriétaire. Dans le second cas, une compagnie de la Couronne, la Wartime Housing Limited, reçoit le mandat de construire des maisonnettes qu'elle offre en location aux ouvriers des usines d'armement (1941-1944) et aux vétérans (1945-1947). Ces politiques fédérales ne durent pas: les logements de la Wartime sont mis en vente dès 1947 et le contrôle des loyers est progressivement délaissé. Elles auront cependant suscité de fortes oppositions et alimenté les débats.

Jusqu'à 1948, les autres paliers de gouvernement évitent d'intervenir. Le seul geste digne de mention est la modification par la Ville

\footnotetext{
7 Choko, Collin et Germain, op. cit.
} 
de Montréal des règlements de zonage de quelques quartiers pour permettre la construction des maisonnettes de la Wartime Housing Limited et celle des bungalows en général. Cet immobilisme n'est pas étonnant car, au Québec, les forces sociales opposées à l'intervention de l'État, dans le logement comme dans l'ensemble des conditions de vie, sont importantes et jouissent d'un poids politique majeur.

C'est particulièrement le cas des organisations catholiques dont la stratégie d'action est orientée vers la promotion pour le Québec d'un modèle corporatiste de société, inspiré de la doctrine sociale catholique ${ }^{8}$. Elles veulent que l'État, à tous les niveaux, se confine à un rôle plus effacé, moins dirigiste que celui que cherchent à se tailler l'Administration nationale du logement puis la Société centrale d'hypothèques et de logement, organismes chargés de l'application des politiques fédérales. Par ailleurs, proches des courants nationalistes québécois, ces organisations préfèrent que l'intervention étatique passe par le gouvernement provincial et non par Ottawa. La solution aux problèmes du logement doit venir, en priorité et pour l'essentiel, de la société civile. C'est ce que la Ligue ouvrière catholique (LOC) va tenter de démontrer et d'imposer.

\section{A CHAQUE FAMILLE, SA MAISON: LES LIGNES DE FORCE DU PROJET D'ACTION CATHOLIQUE}

Fondée en juillet 1939, la LOC se place, entre 1943 et 1951, au centre d'une importante campagne de revendications pour l'amélioration des conditions d'habitation en milieu urbain. Le thème majeur consiste à exiger du gouvernement provincial qu'il instaure le «crédit urbain», c'est-à-dire la remise d'une portion de l'intérêt, de manière à ramener le taux d'intérêt hypothécaire, pour l'emprunteur, à 2,5\%. En complément, les gouvernements devraient garantir un prêt équivalent à $100 \%$ de la valeur de la maison, jusqu'à un certain plafond.

Fort critique des actions du gouvernement fédéral et de l'inaction du gouvernement provincial et des municipalités, la Ligue ouvrière catholique mène plusieurs enquêtes dont elle rapporte les résultats dans son journal hebdomadaire, Le Front ouvrier. Le tableau est sombre: logements exigus, entassement à plusieurs familles, insalubrité, cherté des logements et marché noir, faible taux de propriétaires... En conclusion de ses critiques et de ses constats, la Ligue avance le slogan «A chaque famille, sa maison» et réclame une action significative de la part des autorités gouvernementales (voir illustration).

Les mesures qui sont réclamées de Québec - le crédit urbain et l'annulation, à certaines conditions, du versement initial — visent à

8 Clinton Archibald, Un Québec corporatiste? (Hull, Les Éditions Asticou, 1984), 429 p. 


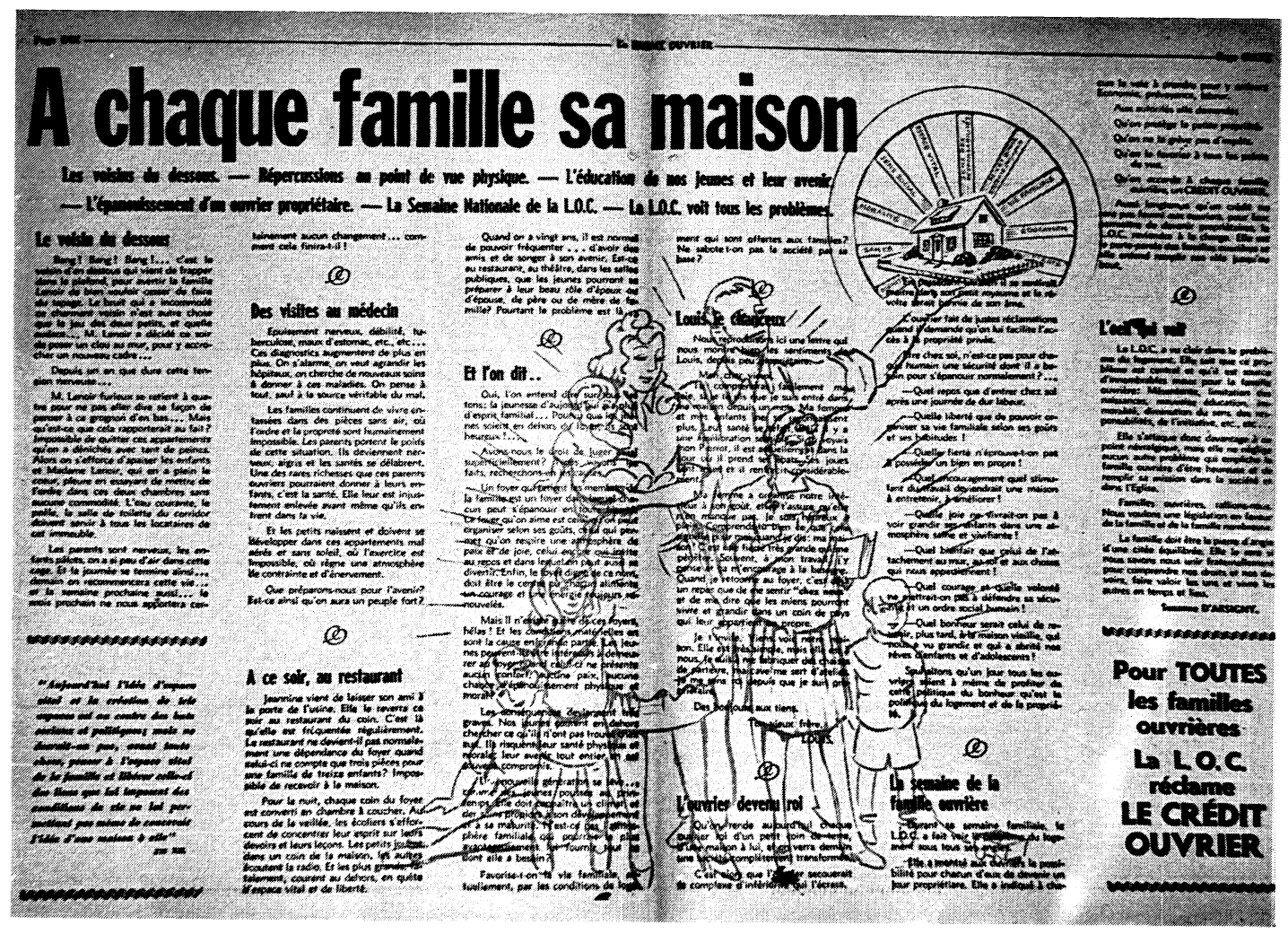

Cet extrait de l'hebdomadaire de la LOC est révélateur de la vision du logement ouvrier idéal et des stratégies d'action de la Ligue (Le Front ouvrier, 3,26 bis, mai 1947).

faciliter l'accès à la propriété de maisons unifamiliales modestes bien que confortables pour les ouvriers (et les gagne-petits). Les municipalités doivent aussi contribuer à l'effort. On réclame d'elles qu'elles cèdent à un prix symbolique les lots à bâtir qui sont en leur possession et qu'elles octroient une remise partielle des taxes foncières pour une certaine période.

La plupart des organisations canadiennes-françaises appuient les demandes de la LOC. Mentionnons le mouvement coopératif, l'Église catholique, la Confédération des travailleurs catholiques du Canada (CTCC), les Sociétés Saint-Jean-Baptiste, le Bloc Populaire, et même certains éléments de la Chambre de commerce de Montréal. Les pro- 
positions d'action ne restent pas sans lendemain puisque le gouvernement de Duplessis adopte, au début de 1948, une version mitigée du crédit urbain avec la loi «pour améliorer les conditions de l'habitation» qui accorde, à certaines conditions, une bonification d'intérêts de 3\% pour des prêts hypothécaires d'au plus $6000 \$$. Ce programme de rabais d'intérêt, en vigueur jusqu'en 1975, est administré par l'Office du crédit agricole du Québec.

De même, au printemps de 1948, la Ville de Montréal y va de ses règlements «pour remédier à la crise du logement». Si le propriétaire habite l'immeuble, terminé dans un délai prédéterminé, et si l'évaluation imposable est en deçà d'un certain seuil, le propriétaire, comme individu ou comme membre d'une coopérative d'habitation, peut bénéficier d'une prime maximale de 299 \$ sur l'achat d'un lot à bâtir acquis de la municipalité. En outre, il peut bénéficier d'une remise de $50 \%$ des taxes foncières générales pendant quinze ans. En pratique, la Ville de Montréal a surtout cherché à se départir des nombreux lots acquis pour défaut de paiement des taxes foncières lors de la crise des années trente. L'application des règlements n'a pas l'impact attendu par les groupes coopératifs. Il n'y eut que 2345 bénéficiaires de primes de 289 \$ en moyenne pour l'achat de lots vendus souvent à $300 \$$ par la municipalité .

Mais, pour les promoteurs du crédit urbain, l'action de l'État a un rôle strictement supplétif. Elle a pour but de créer les conditions favorables à une solution au problème de l'habitation. Il ne revient, en aucun cas, à l'État de régler seul les problèmes du logement. La responsabilité première en incombe aux ouvriers eux-mêmes. Le rôle de l'État est de soutenir l'action de ceux qui savent s'aider, de prendre le parti des «ouvriers méritants», c'est-à-dire ceux qui sont bons pères de familles, d'une part, et qui n'hésitent pas à faire l'effort d'épargner, de bâtir en corvée ou de participer aux cercles d'étude. Dans la conception des animateurs de l'action catholique, le principal moyen à la disposition de l'ouvrier et du salarié c'est la coopération. Parallèlement à l'action revendicatrice, des efforts sont donc déployés pour faire surgir des coopératives d'habitation dans la plupart des centres urbains québécois.

Entre 1941 et 1965, près de 200 coopératives d'habitation ont été fondées. Par diverses stratégies d'action, ces coopératives ont contribué, avec plus ou moins d'intensité et avec une importance variable d'une région du Québec à l'autre, à la construction d'environ 10000 maisons. En générai, ces maisons sont de type unifamilial mais certaines comportent deux logis - le second étant destiné à la location. La période 1954-1960 a été la plus active avec, pour l'ensemble du

9 Ville de Montréal, Rapport du directeur du service des finances, pour les années budgétaires 1948-1949 à 1955-1956. Compilations de l'auteur. 
Québec, une production annuelle moyenne supérieure à 750 nouvelles maisons. Avant 1949 et après 1966, ces coopératives ont joué un rôle insignifiant. Entre ces deux dates, leur production équivaut à 1,6\% de la construction neuve au Québec ${ }^{10}$. C'est peu, encore que cette performance soit trois fois supérieure à celle des coopératives ailleurs au Canada.

Cependant l'impact social des coopératives d'habitation de cette période ne doit pas être négligé. Ce ne sont que 10000 nouveaux logements. Mais ce sont 10000 nouveaux propriétaires dans une province où, en 1961 , les ménages urbains sont encore à $60 \%$ locataires. Avec l'option pour l'action coopérative, les mouvements d'action catholique ne veulent pas seulement instituer un mécanisme capable de produire des logements. Ils veulent rendre propriétaires les familles ouvrières et salariées. Pour y arriver, deux grands types de syndicats coopératifs de construction d'habitation ont été mis à l'épreuve.

La formule des «coopératives de bâtisseurs» reprend d'assez près un modèle mis au point quelques années plus tôt par des militants catholiques du mouvement Antigonish dans la province de la NouvelleÉcosse $^{11}$. Avant la construction des maisons, il y a une phase, souvent longue, au cours de laquelle les sociétaires participent à un programme d'épargne volontaire et se réunissent en cercle d'étude. L'organisation est artisanale et la capacité de production est presque toujours réduite. Généralement, la coopérative n'a qu'un objectif: faire accéder à la propriété un nombre restreint de membres. Elle ne survit pas à cet objectif. Les coopératives de bâtisseurs se distinguent entre elles par l'importance du travail en corvée auquel elles recourent. A un extrême, le sociétaire ne devient propriétaire que le jour où le groupe a complété la construction de toutes les maisons. A l'autre extrême, chaque sociétaire construit seul sa maison et l'association coopérative n'est utile que pour l'achat du terrain, l'énoncé de certaines règles d'urbanisme et, peutêtre, l'accès aux institutions financières coopératives. Néanmoins, toutes ces coopératives reposent sur la corvée ou l'autoconstruction pour réduire les coûts.

La formule des «coopératives d'accession à la propriété» est plus tardive. Elle a été mise au point par le Service de l'Habitation Ouvrière fondé à Drummondville en 1947. Toute forme de corvée ou d'autoconstruction n'est pas, au départ, exclue. Mais il y a dans cette seconde formule une recherche de professionnalisme, comme stratégie de réduction des coûts. La base fonctionnelle de ces coopératives consiste à rassembler le pouvoir d'achat et le pouvoir d'emprunt d'un nombre

\footnotetext{
10 Jean-Pierre Collin, La Cité coopérative canadienne-française: Saint-Léonard-de-PortMaurice, 1955-1963 (Montréal, Les Presses de l'Université du Québec, 1986), 184 p.

11 Francis J. Mifflen, The Antigonish Movement: A Revitalization Movement in Eastern Nova Scotia. Thèse de doctorat en sociologie au Boston College, Boston, 1974, 233 p.
} 
suffisamment grand de coopérateurs pour faire construire des maisons à bon compte, par un entrepreneur si nécessaire, mais idéalement par ses propres ouvriers. Ces coopératives cherchent à atteindre une clientèle plus large et visent la permanence de l'organisation. C'est cette seconde formule qui a permis au mouvement d'effectuer une percée dans les milieux fortement urbanisés, tout particulièrement à Montréal, comme nous le verrons plus loin.

En majorité, les coopératives de l'un ou l'autre type ont été fondées par des membres de l'Église catholique: des curés dans plusieurs paroisses, mais surtout des militants de la Ligue ouvrière catholique. Ces derniers ont aussi inspiré et fortement contribué au regroupement des coopératives dans une fédération provinciale. En effet, en 1947, les coopératives d'habitation liées à la LOC se rassemblent à Montréal pour fonder la Commission nationale de l'habitation. Cette Commission prépare la voie à la Fédération des coopératives d'habitation du Québec, dont le congrès de fondation se déroule à Trois-Rivières à l'automne 1948. La Fédération se définit comme un organisme d'information, d'éducation et de représentation, comme une structure de liaison avec le reste du mouvement coopératif et comme une centrale d'achat des matériaux de construction. La plupart des coopératives d'habitation ont adhéré à la Fédération qui, malgré ses nombreuses crises internes, a certainement contribué à insuffler au mouvement une vigueur plus forte que dans les autres provinces canadiennes.

L'action des coopératives d'habitation, individuellement ou en Fédération, vise à procurer sa maison à chaque famille ouvrière. Mais «l'espace vital» de la famille ouvrière ne peut pas venir de n'importe quelle sorte de logements. Inspirés à la fois par les discours des hygiénistes réformistes du début du siècle, par les thèses de certains urbanistes et architectes et par la morale familiale tirée des encycliques papales, les idéologues du mouvement s'attardent à définir les caractéristiques générales et détaillées du logement idéal, en regard de l'objectif de répondre à l'espace vital de la famille ouvrière et de son chef. De cet examen, ils concluent que la «maison», c'est-à-dire l'unifamiliale détachée de toute autre construction, en accession à la propriété, est seule capable de répondre adéquatement à toutes et chacune des exigences de base de la maison idéale ${ }^{12}$.

Sans entrer dans les détails, mentionnons que les documents de l'époque ${ }^{13}$ dégagent cinq groupes de critères: 1 ) le logement idéal est

12 Collin, La Cité coopérative, 31-60.

13 Nous pensons ici en particulier aux cahiers de formation en appui aux travaux des cercles d'étude. Par exemple, Habitation a été publié entre 1947 et 1949 par la Commission nationale de l'habitation (animé par la LOC) et $A$ Guide to Co-operative Housing, du St. Patrick's College Institute of Social Action, traduit et adapté vers 1953 et 1954 par la coopérative d'habitation Les Foyers de Ville-Marie. Cette conception de la maison idéale se retrouve aussi dans plusieurs périodiques tels que Le Front ouvrier, Ma Paroisse, Ensemble. 
vaste et salubre; 2) il garantit l'intimité de chacun des membres de la famille; 3) il favorise la stabilité et l'enracinement de la cellule familiale et en protège l'intimité; 4) conformément au besoin naturel inné chez l'homme, il est la propriété du chef de famille; 5) finalement, ce logement idéal s'insère dans un bon voisinage.

Bien sûr, même les coopératives d'habitation sous la gouverne de militants catholiques n'ont pas fait preuve d'une adhésion rigide au modèle idéal. Quelques solutions de compromis sont acceptées. Ainsi, un certain nombre de duplex ${ }^{14}$ et plus rarement des maisons jumelées ont été construites. Ces solutions de rechange ont la plupart des vertus accordées à la maison isolée. Surtout, elles s'inscrivent dans une même famille d'immeubles résidentiels où, au-delà des variations de la densité, chaque logement est une «unité autonome».

Car la promotion de la propriété d'une maison, comme modèle recherché de vie résidentielle, s'effectue aussi en combattant son antithèse: la location dans une conciergerie ${ }^{15}$ et surtout dans une conciergerie avec ascenseur. Le document le plus synthétique sur cette question a été rédigé par un journaliste du quotidien montréalais La Presse, au plus fort de la lutte contre les Habitations Jeanne-Mance, le premier projet de logements publics à Montréal ${ }^{16}$. Ce pamphlet ${ }^{17}$ donne la mesure des préjudices attribués aux immeubles-à-ascenseurs et aux conciergeries en général. Examinons les grandes lignes de ce réquisitoire.

La conciergerie, affirme-t-on, peut être adaptée à certaines catégories de la population: les personnes âgées qui n'ont plus d'enfants avec eux, ou ceux qui n'auront jamais d'enfants. Elle serait, toutefois, en toutes circonstances, inadaptée pour les familles avec enfants et plus particulièrement pour les familles canadiennes-françaises. Ce type de construction, prétend-on, met en danger la santé des individus, l'épanouissement normal de la vie familiale et la liberté de la vie communautaire: autrement dit, il menace l'espace vital de la famille.

Si on admet que, par rapport au taudis, la famille y bénéficie d'une amélioration des conditions d'hygiène et de bien-être, on laisse entendre que l'immeuble-à-ascenseur peut être la source de déséquilibres psychologiques qui introduisent des désordres physiologiques en appa-

\footnotetext{
14 Typique à Montréal, le duplex est un immeuble multifamilial, de moyenne densité qui comprend deux, parfois trois logements répartis sur deux étages. Le duplex se distingue de la conciergerie non seulement par la taille mais aussi par l'individualisation des logements: chacun a un système d'accès individualisé à la rue et il n'existe pas d'équipements ou de services collectifs, même si dans nombre de cas le propriétaire habite l'un des logements.

15 C'est ainsi que l'on désigne à l'époque les immeubles résidentiels à usage locatif.

16 Robert Potvin, Habitations Jeanne-Mance, 1952-1961 (Montréal, Institut d'urbanisme de l'Université de Montréal, 1983), n. p.

17 Ligue d'action civique, L'expérience des autres (Montréal, Brochure no 10 de la Ligue d'action civique, 1957), $61 \mathrm{p}$.
} 
rence (les ulcères d'estomac, les mauvais rhumes, la haute pression artérielle). De plus, par suite de l'usage des corridors et des ascenseurs, les maladies d'enfants s'y propageraient avec une extrême rapidité.

Bref, malpropreté et promiscuité accompagnent, par définition, les escaliers et corridors communs, les laveries et les ascenseurs. On les accuse aussi de causer des désordres moraux. «Les escaliers et les ascenseurs remplacent les petits bois d'il y a 25 ans, pour les expériences amoureuses des adolescents et des plus vieux aussi.» ${ }^{18}$ Les conciergeries constitueraient des milieux propices à l'éclosion des liaisons extra-maritales et les difficultés matrimoniales y seraient plus fréquentes. L'existence d'équipements collectifs et l'entretien du logement et de son environnement par un concierge ou un gérant minent l'intérêt du père pour son logement. Il s'y sent comme un logeur et conséquemment est tenté de déserter femme et famille.

Ce discours tente aussi de faire valoir que, dans les immeubles en hauteur, le taux de criminalité juvénile serait plus élevé que dans l'environnement immédiat. En outre, la mère ne peut pas, du haut de son 6e ou de son 8e étage, surveiller adéquatement ses enfants ali sol, occupée qu'elle est par les tâches ménagères ou les soins au bébé. Il s'ensuit, entre autres, une indépendance trop précoce des enfants. Enfin, la vie communautaire est aussi menacée par le fait que les conciergeries constitueraient un lieu propice à la propagation des idées subversives.

Pour toutes ces raisons, aux yeux de l'action catholique, il faut agir pour que les familles ouvrières deviennent propriétaires d'une «maison à unités autonomes». Puisque le marché privé n'est pas capable de satisfaire ce droit et puisqu'il n'est pas du ressort de l'État de remplir cette mission, il faut s'organiser pour procurer «A chaque famille, sa maison».

\section{LES COOPÉRATIVES D'HABITATION À MONTRÉAL}

L'action coopérative, et partant l'action catholique en matière d'habitation, entre 1940 et 1965, a été traditionnellement associée au milieu semi-rural et à celui des petites villes de province. Si le critère utilisé est celui du nombre de coopératives, cette association est probablement justifiée. Mais, lorsque l'on considère plutôt le nombre de maisons construites, la conclusion diffère. C'est à Trois-Rivières, Québec et Montréal qu'ont émergé les plus grandes coopératives. La région métropolitaine de Montréal a été l'hôte de plus du quart de la production coopérative québécoise.

Il est vrai que l'habitation coopérative a été lente à démarrer dans la métropole. De plus, la difficulté fort répandue de trouver des terrains

$18 \quad$ Ibid., 57. 
à des prix raisonnables a été plus marquée à Montréal que dans les autres centres urbains. Mais la région de Montréal a été le lieu d'expériences remarquables par la taille et innovatrices par les objectifs et par le fonctionnement. Comme ailleurs en province, les militants de l'action catholique ont joué un rôle de premier ordre aux niveaux de la conception, de la direction et de l'animation, dans les grandes comme dans les petites coopératives montréalaises.

L'histoire des coopératives de bâtisseurs et des coopératives d'accession à la propriété à Montréal et dans sa région a cependant été peu fouillée. Les quelques analystes qui se sont intéressés aux coopératives québécoises d'habitation ont ignoré cette région ${ }^{19}$. Plusieurs coopératives mériteraient d'être étudiées en détail. De nombreux aspects du mouvement restent toujours dans l'ombre. La qualité des informations mises au jour varie selon les périodes. Pour toutes ces raisons, l'analyse synthétique du mouvement coopératif d'habitation à Montréal est encore à l'ordre du jour.

Toutefois, dans le cadre de nos recherches sur les enjeux de la transformation de l'espace urbain à Montréal au cours des décennies quarante et cinquante ${ }^{20}$, nous avons eu l'occasion de dessiner la trame générale de ce mouvement. Tout d'abord, nous avons étudié en profondeur deux cas marquants qui balisent le mouvement. Ces monographies ${ }^{21}$ ont porté l'une sur l'Union économique d'habitations, 1940-1947, l'autre sur la Coopérative d'habitation de Montréal, 1955-1963. Ensuite, la consultation systématique des périodiques, publiés par les mouvements d'action catholique, les groupements coopératifs, les centrales syndicales, les organisations nationalistes canadiennes-françaises, nous permet de dresser un tableau préliminaire des coopératives montréalaises entre 1940 et $1965^{22}$.

\footnotetext{
19 Cette remarque s'applique aux travaux les plus récents comme aux plus anciens. Voir C. Vienney, A. Pringent, R. Charbonneau et J.-P. Deslauriers, Analyse socio-économique comparée des coopératives d'habitation en France et au Québec (Chicoutimi, Université du Québec à Chicoutimi, 1985), 391 p.; Hervé Fahndrich, «Une analyse des coopératives de construction au Québec, 1937-1968», Coopératives et Développement, 16,1 (1984): 127-157; Conseil de la coopération du Québec, Les coopératives d'habitation au Québec, Rapport de la Commission de l'habitation du CCQ (Québec, février 1968), 197 p.; César Rutigliano, «Le mouvement des coopératives d'habitation au Québec, 1937-1971», Mémoire de maîtrise en sociologie, Université Laval, 1977), 251 p.; Jacques Godbout, «L'échec des coopératives d'habitation au Québec», L'Habitation et les citoyens, 5,4 (1975): 1-5.

20 Marc-H. Choko, avec la collaboration de Jean-Pierre Collin et Annick Germain, Trans formation de l'espace urbain: rapports de pouvoir et débat social sur les conditions de vie à Montréal, 1940 à nous jours - Rapport de recherche (Montréal, Université du Québec à Montréal, Département de Design), 1985.

${ }_{21}$ Marc-H. Choko, Une Cité-jardin à Montréal: La Cité-jardin du Tricentenaire, 19401947 (Montréal, Université du Québec à Montréal, Département de Design, 1986) et Jean-Pierre Collin, La Cité coopérative.

${ }_{22}$ La liste des articles de périodiques utiles pour dresser ce bilan se trouve dans Jean-Pierre Collin, La cité coopérative, 163-170. Les informations contenues dans ces articles ont été complétées pour certaines, vérifiées pour d'autres à l'aide de dossiers trouvés aux archives du Conseil de la coopération du Québec et aux archives de la LOC conservés par le Mouvement des travailleurs chrétiens de Montréal. Enfin, les statistiques, quant au nombre de maisons construites, sont souvent tirées de Cesar Rutigliano, op. cit.
} 
Peu détaillé, ce bilan manque de profondeur. Néanmoins, il englobe vraisemblablement l'ensemble des coopératives actives au cours de la période ${ }^{23}$. Les oubliés sont les cercles d'étude, les projets qui ont avorté avant la construction d'un premier logement et, peut-être quelques coopératives au rayonnement très discret et à la production très limitée.

L'année d'incorporation, la période principale d'activité, le lieu (quartier ou municipalité) d'opération, le nombre de maisons construites et leur valeur en dollars courants nous sont généralement connus. Parfois, nous pouvons préciser l'origine sociale des fondateurs et des membres, décrire brièvement le mode de fonctionnement, énumérer les difficultés rencontrées et surtout évaluer l'écart entre le projet initial et les réalisations. Finalement, nous avons des indications sérieuses sur le type, le prix et le financement des maisons.

L'analyse de ces données nous amène à distinguer trois phases (tableau 1) dont nous illustrerons les caractéristiques en insistant sur les principales expériences de coopération liées à l'action sociale catholique. Auparavant, il faut s'arrêter à l'histoire d'un précurseur dont l'expérimentation est demeurée isolée: l'Union économique d'habitations (UEH). L'UEH est née, au début de la Deuxième Guerre mondiale, de l'initiative conjointe d'un jésuite et d'un avocat étroitement liés aux organisations catholiques promotrices d'un certain corporatisme social ${ }^{24}$. Après une tentative infructueuse qui avait impliqué les syndicats catholiques de la ville de Québec, Me Gosselin et le père Jean-d'Auteuil Richard se retrouvent à Montréal et fondent l'UEH, en octobre 1940, avec l'appui moral de l'archevêque et des milieux catholiques et avec l'appui financier des Jésuites et des Clercs de SaintViateur. Cette société sans but lucratif entreprend, au début de 1941, la réalisation d'une Cité-jardin du Tricentenaire dans le quartier Rosemont à Montréal (parce que l'année 1942 marque le troisième centenaire de la fondation de Montréal).

D'abord dirigée vers les ouvriers, l'action de l'Union s'adresse finalement à une certaine classe moyenne (celle des voyageurs de commerce, des comptables et des ingénieurs). Ce sont, en particulier, les modifications apportées par le gouvernement fédéral à la Loi nationale sur l'habitation (LNH) qui ont contraint les promoteurs de l'UEH à ce changement de cible. Malgré tout, dans le dossier de la Cité-jardin du Tricentenaire, ils collaborent étroitement avec les hauts fonctionnaires fédéraux qui sont à la recherche d'organisations disposées à mener des expériences pilotes, en vue de préparer le terrain à la production de logements en série après la guerre.

23 Le territoire couvert est la région métropolitaine de recensement, définition de 1961 .

24 Les informations qui suivent sont tirées des archives de l'Union économique d'habitations que nous avons déposées, en collaboration avec Marc H. Choko, au Service des archives de l'Université du Québec à Montréal. 
La Cité-jardin du Tricentenaire tire certains bénéfices de cette association, entre autres, une facilité d'accès au marché hypothécaire et une priorité sur les matériaux de construction, rationnés pendant la guerre. Mais la majorité des coopérateurs n'étant pas pour autant gagnés à l'idée de la collaboration avec l'État fédéral, la Cité-jardin du Tricentenaire s'est avérée un partenaire peu commode, à la limite même encombrant pour les autorités fédérales. La production en série implique que l'on adopte un produit homogène pour une grande diversité d'acheteurs. Or la Cité-jardin regroupe un ensemble très homogène de familles canadiennes-françaises catholiques de la classe moyenne désireuses de se faire bâtir une maison fortement personnalisée ${ }^{25}$ (voir illustration).

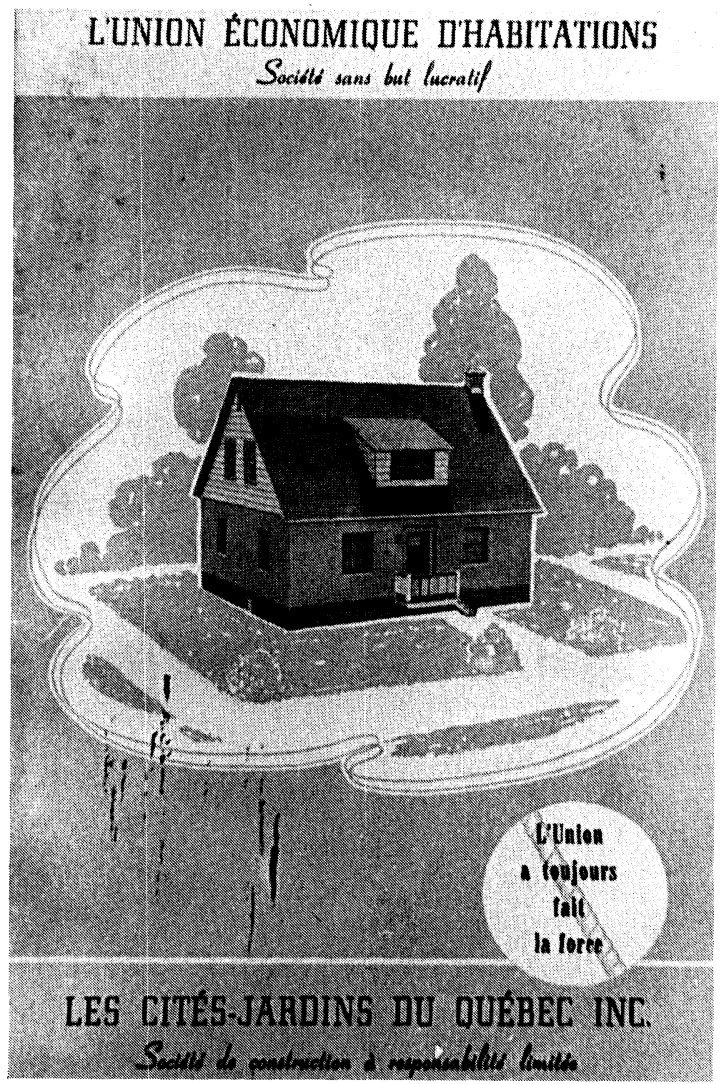

L'UEH a tracé la voie au mouvement coopératif quant au type de logement à produire et quant à l'organisation de la production (Brochure publicitaire de 1943).

25 Choko, Collin et Germain, «Le logement et les enjeux», 131. 
Au plan de l'urbanisme, le projet résidentiel du quartier Rosemont innove puisqu'il représente la première expérience concrète de citéjardin à Montréal. Jusque-là, seuls quelques rares lotissements-jardins pour familles fortunées avaient été tentés et les entreprises de réformes en matière d'urbanisme n'avaient pas donné des résultats très concluants $^{26}$.

La Cité-jardin du Tricentenaire a été un succès mitigé. Entre 1941 et 1947, seulement 167 des 600 maisons unifamiliales du projet initial ont été construites. Pour favoriser l'éclosion d'une réelle communauté nouvelle, le sociétaire devait adhérer à trois coopératives: la coopérative résidentielle, la caisse d'épargne et de crédit et le magasin d'alimentation. L'éclatement de l'UEH en 1946-1947 met fin au rêve communautaire. Les cités-jardins devaient se propager tout autour de Montréal et proliférer en province. Or, un seul autre projet de peu d'envergure a vu le jour à Hull, dans l'ouest de la province de Québec.

Il n'empêche que la Cité-jardin du Tricentenaire a imprimé une direction au mouvement coopératif. Le public visé, en exclusivité ou presque, ce sont les familles nucléaires canadiennes-françaises et catholiques qu'il faut rendre propriétaires. Selon les époques et les projets, l'on s'adresse tantôt aux ouvriers, tantôt à la classe moyenne et parfois même aux deux groupes. Un type de logement est considéré supérieur à tous les autres: la maison unifamiliale. Enfin, quelques autres coopératives montréalaises tentent de dépasser le problème du logement pour générer des communautés modèles.

D'abord bien accueillie par le milieu coopératif québécois, l'UEH a mauvaise presse à partir de 1944, après qu'elle eût tenté d'inféoder les autres coopératives d'habitation par une structure pseudo-fédérative. A partir de ce moment, l'interprétation officielle accorde le statut de pionniers à des groupements coopératifs beaucoup plus modestes et aux ambitions moins généreuses: les coopératives de bâtisseurs.

En effet, entre 1945 et 1948, se créent des coopératives inspirées du modèle apparu dans plusieurs agglomérations de taille moyenne (Saint-Jean, Chicoutimi, Victoriaville). Ces coopératives, préoccupées de loger les ouvriers à bon compte, font largement appel au travail en corvée pour réduire le coût de production des maisons. Bien que certaines furent théoriquement actives jusqu'en 1952, elles n'ont collectivement construit qu'une centaine de maisons. Ces coopératives ont systématiquement failli à la tâche, les réalisations étant toujours minimes en regard des objectifs initiaux.

Il en va ainsi de la Coopérative d'habitation de Jésus-Ouvrier. Fondée à Longueuil en 1947 par des militants de la Ligue ouvrière

\footnotetext{
${ }^{26}$ Jeanne M. Wolfe, The Progress of Housing Reform: Three Early Experiments in Montreal (Montréal, Université McGill, School of Urban Planning, 1981), 26 p.
} 
catholique (LOC), elle aurait regroupé, dans des cercles d'études, 281 membres au moment de son incorporation. La production s'arrête toutefois à 21 maisons unifamiliales, en 1952. La coopérative, qui fait appel à la corvée mais ne parvient pas à se financer suffisamment, déclare regrouper encore 150 membres à cette date. Elle n'a toutefois produit aucun autre logement.

Les syndicats catholiques y vont aussi de leur expérimentation coopérative et, entre 1947 et 1949 , le Conseil central des syndicats nationaux de Montréal entreprend la construction de maisons unifamiliales dans le quartier montréalais de Cartierville. Toutefois, après en avoir bâti quarante, l'organisme syndical met un terme à l'expérience puisque, avec l'importance de la mise de fonds exigée, seulement trois syndiqués ont pu profiter de ce programme d'accès à la propriété. Le Conseil central aura tout de même innové en construisant des maisons jumelées. Quelques autres coopératives montréalaises ont repris ce modèle par la suite. Ailleurs au Québec, les coopératives d'habitation n'y ont recours qu'après 1962 .

La paroisse s'impose aussi comme un lieu d'expérimentation. Dans le quartier Émard, l'Unité des Ormeaux qui exploite un magasin coopératif depuis 1944 ajoute le logement à ses activités. Une cinquantaine de chefs de famille participent aux cercles d'étude et en 1950, vingtdeux familles habitent leur nouveau logement, bâti en corvée. Pour y arriver l'Unité des Ormeaux a aussi tiré profit de la vente par la Ville de Montréal de lots à bon marché. La mise en vigueur des règlements 1881 et 1882 de la municipalité favorise d'ailleurs l'émergence d'un nouveau genre de coopératives d'habitation particulier à la région de Montréal.

A la phase des coopératives de bâtisseurs, dont on a ici trois exemples, succède, en effet, la phase des coopératives de services techniques en matière d'habitation. Ainsi, à partir de l'été 1948, au moment où se crée la Fédération, les coopératives montréalaises se structurent sur d'autres bases. Elles n'ont le plus souvent qu'un rôle d'intermédiaire entre l'individu futur propriétaire de son logis, et le constructeur. Bien qu'elles aient supervisé la construction d'un nombre respectable de logements (environ 900), ces coopératives n'ont qu'une influence limitée sur les coûts de construction des dits logements et regroupent essentiellement des ménages de la classe moyenne.

Il y a d'abord une dizaine de petites coopératives regroupant de dix à quinze familles d'une même paroisse catholique. Fondées entre 1949 et 1952, le plus souvent par des militants de la LOC, ces coopératives paroissiales, dont certaines font construire des duplex plutôt que des maisons unifamiliales, copient les objectifs et la manière de faire du Comité d'habitation de Montréal (voir illustration). 


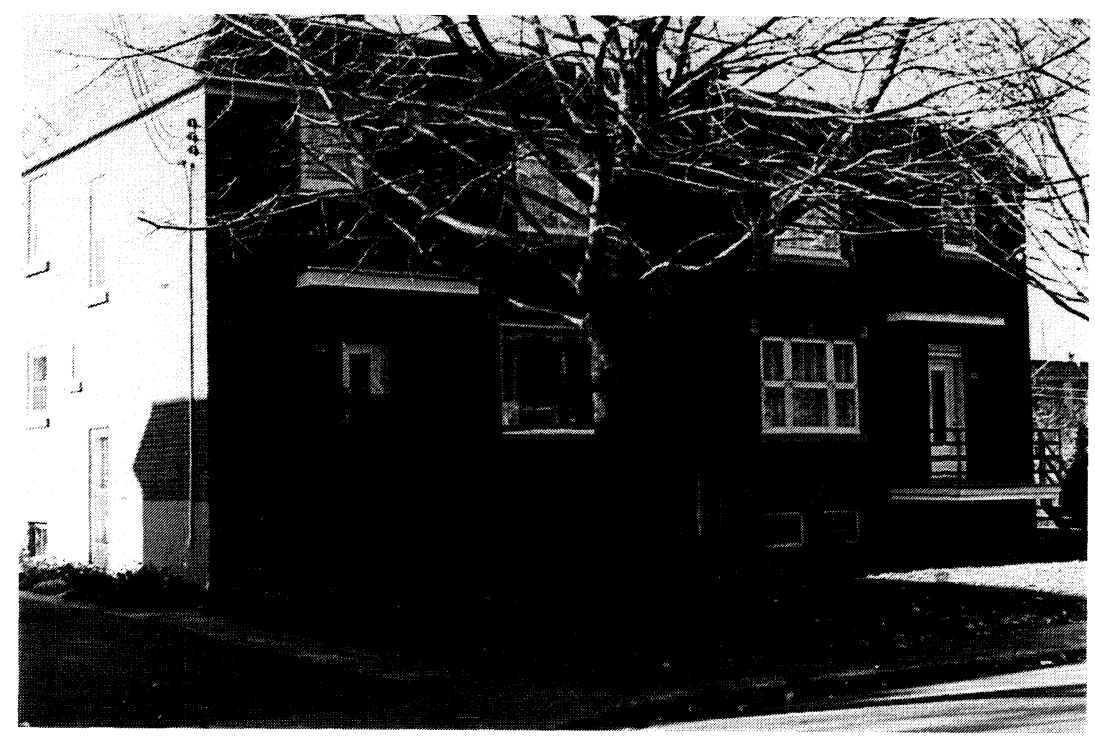

Les maisons du Comité d'habitation de Montréal de la LOC sont généralement des unifamiliales jumelées de deux étages (Quartier Mercier à Montréal, novembre 1984).

Ce syndicat coopératif de construction a été fondé, en avril 1948, par et pour les militants et adhérents de la Ligue ouvrière catholique à Montréal afin de profiter, de manière organisée, des programmes de subventions à l'accession à la propriété, adoptés la même année par la Ville de Montréal. Le Comité d'habitation de Montréal n'a qu'un rôle de mandataire coordonnateur. Il recherche les lots à bâtir et les prêteurs hypothécaires et il conçoit des plans de maisons. Cependant, le Comité n'intervient pas dans la construction comme telle des maisons. Cette partie de l'opération est confiée à un entrepreneur général qui, à son tour, fait appel à des sous-traitants. Tous ces entrepreneurs acceptent de ne prendre pour leurs travaux qu'une marge limitée de profit.

Malgré un départ encourageant, le Comité d'habitation de Montréal est resté loin de son objectif initial de 1500 maisons et, entre 1948 et 1950 , ne supervise la construction que de 540 maisons unifamiliales jumelées de deux étages dans les quartiers Ahuntsic, Mercier et Rosemont. Contrairement aux intentions du début, l'action du Comité rejoint peu les ouvriers au revenu modeste. Cela est confirmé par le fait que la majorité des coopérateurs ne sont finalement pas éligibles aux subventions municipales parce que leur maison est jugée trop «luxueuse». 
Le rôle effacé de la coopérative d'habitation dans la construction des logements et son orientation vers la classe moyenne sont encore plus évidents dans les années 1952-1955 lorsqu'aux coopératives paroissiales d'une quinzaine de membres succèdent des coopératives de banlieue à peine plus importantes par la taille. Les fonctions de services techniques exercées par le syndicat coopératif s'adressent essentiellement au cadre à l'intérieur duquel la construction s'effectue. L'extrait qui suit résume le rôle exercé par ce genre de coopératives.

La formule du Syndicat coopératif d'habitation de Beloeil offre à ses membres: 1) un centre domiciliaire moderne où sont mises à profit les dernières données de l'urbanisme; 2) des services publics (rue, aqueduc, égouts collecteurs); 3) une centralisation des loisirs; 4) une précieuse assistance dans les pourparlers avec l'entrepreneur, les compagnies prêteuses et la Société centrale d'hypothèques; 5) un comité consultatif d'urbanisme composé d'un architecte et d'un ingénieur ${ }^{27}$.

Les militants de l'action catholique ne semblent pas avoir été très présents dans ces entreprises banlieusardes. On les retrouve plutôt dans des projets parallèles qui visent à ramener le mouvement coopératif dans la ville centrale et à favoriser un retour à la clientèle ouvrière ou de faible revenu.

Inspiré par la maison modèle de Sainte-Monique-les-Saules, construite par les syndiqués en banlieue de la ville de Québec, le conseil central des syndicats catholiques de Montréal, à l'été de 1953, renoue avec le projet de réaliser des maisons coopératives. La construction tarde toutefois à démarrer. De leur côté, les coopératives paroissiales piétinent et cherchent encore une formule susceptible d'assurer le succès de leurs projets domiciliaires. Pour certains, l'action doit dépasser le territoire paroissial. C'est le cas des Foyers de Ville-Marie qui regroupe les animateurs du Service de préparation au mariage. Les Foyers sont incorporés en 1954 comme coopérative d'habitation opérant à l'échelle de la ville de Montréal.

Les Foyers de Ville-Marie jouent un rôle important dans le rassemblement, en 1955, des syndicats catholiques, des coopératives paroissiales et de diverses autres organisations coopératives et sociales. Ce regroupement des maigres avoirs et surtout des énergies inaugure la troisième phase de l'action coopérative à Montréal. En effet, la fondation de la Coopérative d'habitation de Montréal amène le mouvement à intervenir plus intensément dans la construction des maisons et à professionnaliser son action.

Comme on le voit au tableau 1, les coopératives d'accès à la propriété ont été les plus productives avec plus de 1500 maisons construites

\footnotetext{
27 Marcel Blouin, «La cité parfaite. Quelques utopistes réalisent leur rêve au vaste domaine de Pré-Vert, près de Beloeil», La Patrie, 2 octobre 1955, 99.
} 
entre 1955 et 1965 . Au cours de ces dix années, la Coopérative d'habitation de Montréal est le chef de file incontestable qui inspire les autres coopératives de la région ${ }^{28}$. Au plan de l'organisation, elle est soutenue par la Fédération des coopératives d'habitation du Québec. Elle est fortement épaulée par les institutions financières du mouvement coopératif canadien-français. L'époque de la corvée et celle du recours à un entrepreneur général privé sont révolues. La coopérative d'habitation est dorénavant voulue comme un instrument de la prise en charge économique des Canadiens français.

\section{TABLEAU 1}

Bilan de la production domiciliaire par les coopératives d'habitation de la région de Montréal*, 1940-1965, (nombre de maisons)

\begin{tabular}{lllr}
\hline Un précurseur & $1940-1947$ & $\begin{array}{l}\text { La Cité-jardin du } \\
\text { Tricentenaire }\end{array}$ & 167 \\
\hline $\begin{array}{l}\text { Les coopératives } \\
\text { de bâtisseurs }\end{array}$ & $1945-1952$ & $\begin{array}{l}\text { La Coopérative } \\
\text { d'habitation de }\end{array}$ & 21 \\
& & $\begin{array}{l}\text { Jésus-Ouvrier } \\
\text { Le Conseil central } \\
\text { des syndicats nationaux } \\
\text { L'Unité des Ormeaux }\end{array}$ & 40 \\
& & Autres coopératives (3) & 22 \\
\hline $\begin{array}{l}\text { Les coopératives } \\
\text { de services } \\
\text { techniques }\end{array}$ & $1948-1958$ & $\begin{array}{l}\text { Le Comité d'habitation } \\
\text { de Montréal }\end{array}$ & 540 \\
\hline $\begin{array}{l}\text { Les coopératives } \\
\text { d'accession à la } \\
\text { propriété }\end{array}$ & & Autres coopératives (12) & 373 \\
\hline & $1955-1965$ & $\begin{array}{l}\text { La Coopérative } \\
\text { d'habitation de Montréal } \\
\text { Autres coopératives (5) }\end{array}$ & 948 \\
\hline
\end{tabular}

* Selon la définition du recensement fédéral de 1961.

Bien que, comme celles des années 1945-1955, les autres coopératives de la période furent circonscrites dans l'espace et limitées à un groupe restreint de membres, la Coopérative d'habitation de Montréal a, elle, des ambitions métropolitaines (voir carte). Elle compte bâtir des maisons pour des groupes successifs de 200 à 300 membres. Son pre-

${ }^{28}$ La Coopérative d'habitation de Montréal est aussi le modèle suivi par une nouvelle génération de coopératives qui se créent dans les centres urbains québécois. Ces coopératives dominent totalement la Fédération après 1960. Voir Collin, La Cité coopérative, chapitre 5. 


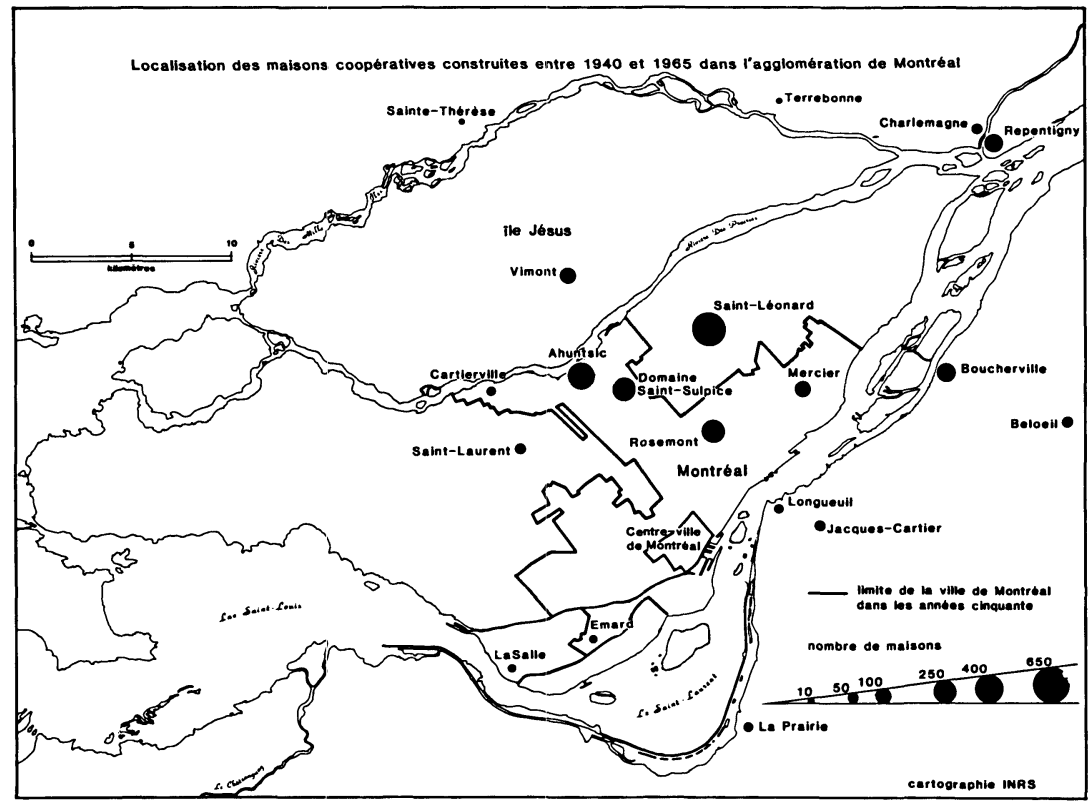

mier terrain d'expérimentation est à Saint-Léonard-de-Port-Maurice, en banlieue de Montréal, où elle a acquis une importante réserve foncière ( 78 hectares). Dès 1956 , la Coopérative embauche, à temps plein, un gérant, du personnel clérical et une équipe d'ouvriers de la construction; elle possède la machinerie nécessaire à l'opération d'un chantier et elle se dote d'un entrepôt.

La Coopérative d'habitation reprend donc le projet, mis en veilleuse depuis la liquidation de l'Union économique d'habitations en 1947, de bâtir sur des bases professionnelles une organisation coopérative permanente dans le secteur de la construction domiciliaire. Comme à la Cité-jardin du Tricentenaire, l'on conçoit que l'action coopérative ne doit pas se limiter strictement au logement mais s'adresser à l'ensemble des conditions de la vie urbaine. Les animateurs de la Coopérative d'habitation de Montréal cherchent donc à faire surgir une communauté modèle qui concrétise en milieu urbain, les valeurs de la morale chrétienne et l'émancipation économique des Canadiens français.

Cependant, au contraire de ceux de l'UEH, les dirigeants de la Coopérative d'habitation de Montréal mènent leur entreprise sans s'inscrire dans la stratégie fédérale d'intervention dans le domaine du logement. Ils sont fidèles à l'opposition traditionnelle des milieux catholiques et nationalistes canadiens-français face à l'État-providence. Ils 
n'acceptent qu'une aide circonstanciée et légère de la part du gouvernement provincial. C'est seulement, après 1960, sous la contrainte des nouvelles règles de financement hypothécaire que la Coopérative finit par avoir recours aux dispositions de la Loi nationale sur l'habitation.

Construire, en banlieue de Montréal, 654 maisons unifamiliales, conformes aux pratiques de l'époque quant à la dimension et à la qualité des maisons, constitue, en soi, une réussite exceptionnelle (voir illustrations). D'autant plus que ces maisons sont remises au propriétairesociétaire à des prix avantageux et avec des conditions rares d'accès au financement hypothécaire personnel. Mais aussi, la Coopérative d'habitation de Montréal a atteint de manière appréciable son objectif de rejoindre la «classe salariée» au sens large.

En effet, les cols bleus, pour la plupart dans des secteurs industriels fortement syndiqués, comptent $40,5 \%$ des chefs de famille et les cols blancs et employés de services privés et publics, un autre $34,5 \%$. Le dernier bloc de $22 \%$ est composé de cadres intermédiaires, de techniciens, de vendeurs à commission et de semi-professionnels selon les normes de l'époque (v.g. instituteurs). Les catégories du bas de l'échelle hiérarchique des professions (v.g. journaliers) sont peu nombreuses mais les cadres supérieurs, les entrepreneurs, les commerçants et les membres des professions libérales sont presqu'absents. Précédemment locataires dans la ville de Montréal, ces jeunes chefs de famille canadiens-français (35 ans ou moins) comptent sur un revenu stable quoique modéré et nourrissent un espoir de promotion sociale à court et à moyen terme.

Cependant, le succès de la Coopérative s'est limité au premier chantier domiciliaire de Saint-Léonard-de-Port-Maurice. Au début des années soixante, le marché du logement ne connaît plus les difficultés et les ratés de la période 1940-1955. Les conditions de financement, garanties par l'État, sont plus généreuses et l'accès à la propriété est facilité. L'heure est au logement social pour les plus démunis de la société. Tous ces facteurs s'ajoutent aux difficultés d'organisation interne et aux erreurs administratives pour conduire à l'échec des chantiers coopératifs à Charlemagne, à Ville Jacques-Cartier et dans Montréal même (au Domaine Saint-Sulpice). A Saint-Léonard même, le projet de cité coopérative canadienne-française perd progressivement sa force mobilisatrice. L'ensemble du mouvement coopératif d'habitation doit d'ailleurs se remettre en cause à partir de 1962. Une réorganisation en coopératives de location, dans des ensembles multifamiliaux, intervient, finalement, en 1967, avec la fondation de Coop Habitat ${ }^{29}$.

\footnotetext{
29 Godbout, op. cit.
} 

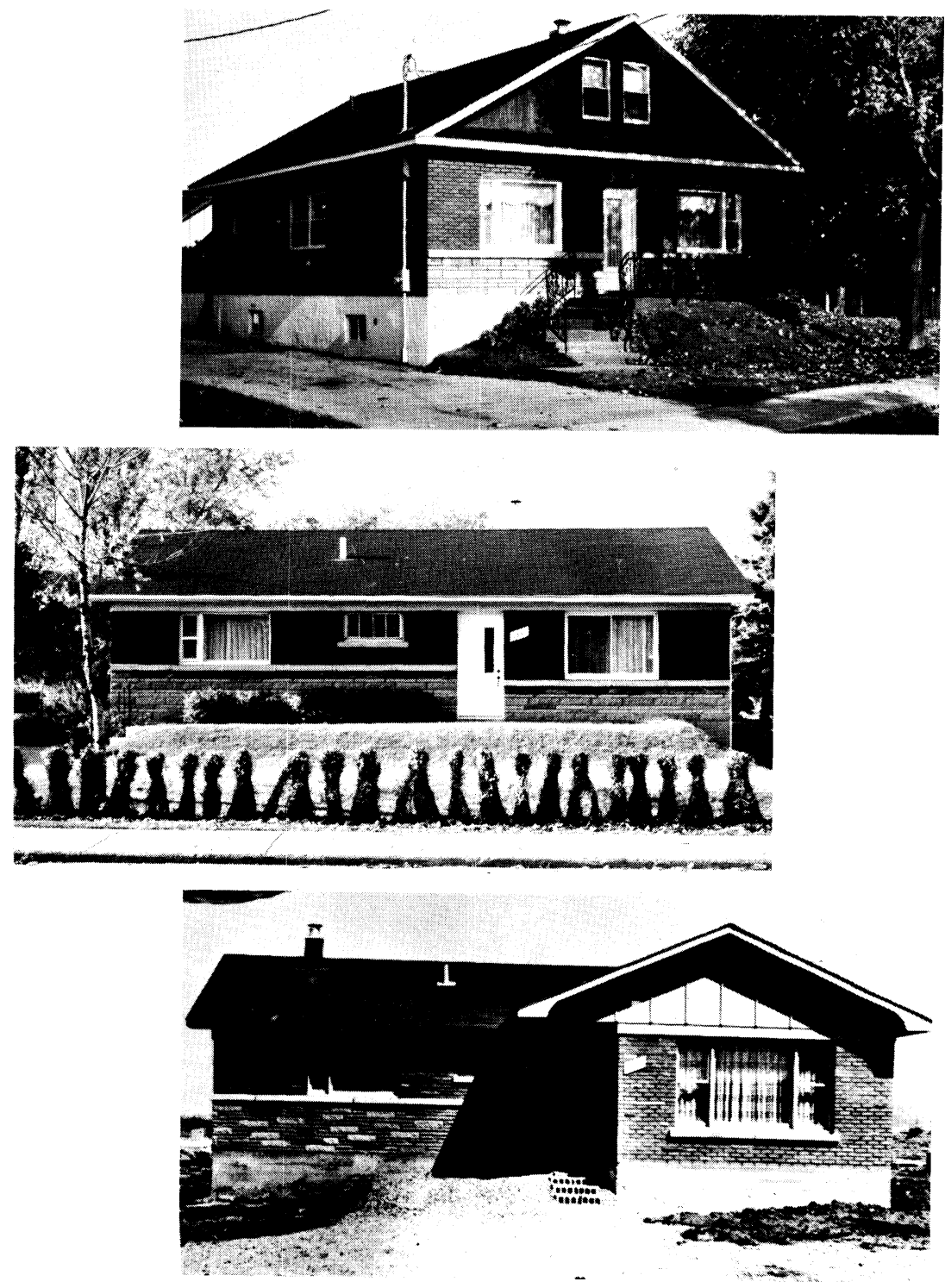

Quelques maisons de la Coopérative d'habitation de Montréal. La maison à un étage et demi (photo du haut) a été publicisée comme celle de la famille nombreuse. Ce sont toutefois les modèles bungalow simple (photo du centre) et bungalow en L (photo du bas) qui ont été les plus demandés par les coopérateurs. 


\section{CONCLUSION}

Quel bilan doit-on tirer des stratégies des mouvements d'action catholique, en regard de la crise du logement à Montréal durant et après la Deuxième Guerre mondiale? Il faut, en premier lieu, évaluer l'apport de l'action catholique au volume de la production domiciliaire et à l'émergence d'un nouveau modèle résidentiel. En second lieu, il faut souligner l'insertion de cet apport dans l'évolution de la prise en charge collective des problèmes sociaux.

Les interventions provinciales et municipales dans le domaine du logement sont largement le résultat de la campagne d'opinion pour le «crédit urbain». En outre, même si les ouvriers et les petits salariés n'en furent pas toujours les bénéficiaires, les réalisations coopératives ( 2500 maisons) sont importantes. Ce nombre, à première vue faible, doit être placé dans son contexte.

Contrairement à l'impression très répandue (même chez les spécialistes), la période 1945-1960, à Montréal, marque seulement le démarrage de la production massive de maisons unifamiliales. Ce type de logements ne domine pas encore le marché. De fait, les années cinquante sont plutôt celles d'une explosion de la construction de logements locatifs ${ }^{30}$. A Montréal, comme dans la région de Québec ${ }^{31}$, prolifère un modèle de conciergeries sans ascenseur de petite taille, visiblement inspiré des expériences de Housing Entreprise Limited, qui remplace le modèle du triplex ${ }^{32}$. La domination de l'unifamiliale s'affirme après 1960 et ce n'est que dans les années 1972-1976 que ce genre de logements est majoritaire dans la production neuve.

La propriété de la maison unifamiliale en banlieue: ce modèle résidentiel proposé par l'action catholique n'est évidemment pas très original dans une Amérique du Nord qui vit à l'heure de la banlieue et de la culture du «drive-in»" 33 . Mais cette action n'est pas pour autant le simple reflet de la mode du temps. Dans le contexte montréalais, où le statut de locataire est la règle, les organisations d'action catholique s'inscrivent parmi les principaux promoteurs d'un modèle résidentiel

30 Georges Mathews, Évolution générale du marché du logement de la région de Montréal de 1951 à 1976: données synthétiques sur une réussite méconnue (Montréal, INRS-Urbanisation, Études et documents no 17, 1980), 69 p. et Marc-H. Choko, Evaluation of Rental Housing Market Problems: Montreal as a Case-Study, 1825-1986 (Montréal, Université du Québec à Montréal, miméo, 1986).

31 Gérald Fortin, «Une ville américaine moyenne, unique en son genre», Recherches sociographiques, 23,2 (1981): 188-203.

32 Le triplex partage les caractéristiques du duplex. Il comprend, sur trois étages, de trois à six logements: ce qui lui confère un caractère locatif plus prononcé. Voir INRS-Urbanisation, La dynamique des espaces résidentiels, texte à l'appui du Colloque international sur la dynamique des espaces résidentiels, (Val Morin, Québec, juin 1982), n. p.

Kenneth T. Jackson, Crabgrass Frontier: The Suburbanization of the United States (New York, Oxford University Press, 1985). 
lié à un nouveau mode de vie ${ }^{34}$ chez la classe des salariés prise au sens large et, notamment, chez les ouvriers.

Avec ses coopératives de construction d'unifamiliales pour sociétaires-propriétaires, l'action catholique à Montréal est donc à la fois le produit du contexte économique et un agent actif de définition de ce contexte. Cette dualité se retrouve dans l'évaluation de la portée sociopolitique de l'action catholique en habitation.

Avec sa notion d'ouvrier méritant, l'action catholique (et coopérative) a une saveur conservatrice indéniable. En outre, elle paraît régressive puisque, concrètement, ses solutions n'englobent pas les problèmes des plus démunis. Dans les décennies quarante et cinquante, l'action catholique est résolument fermée aux initiatives de type socialdémocrate (même celles que les libéraux reprennent à leur compte) qui cherchent à mettre la collectivité plutôt que la famille et l'individu au coeur de la solution ${ }^{35}$. Comparativement aux coopératives new-yorkaises dont l'action repose sur la formation de collectivités nouvelles imbues des valeurs communautaires et dont les immeubles en propriété collective sont soustraits des mécanismes ordinaires du marchép ${ }^{36}$, les coopératives montréalaises offrent un produit traditionnel et s'insèrent volontiers dans les mécanismes normaux du marché capitaliste du logement. D'ailleurs, elles n'amorcent aucune critique de fond du marché capitaliste et se contentent de dénoncer les abus du système; plus précisément les écarts de conduite des trusts ${ }^{37}$.

Mais les résultats de l'action catholique ne sont pas en toutes circonstances rétrogrades. Au contraire, il y a eu, à plusieurs reprises, recherche d'innovations: mise en pratique du concept de cité-jardin ou adhésion aux crédos de l'urbanisme fonctionnel; volonté de faire émerger, par la coopération et dans le respect de la primauté de la famille, des communautés modèles adaptées à la réalité urbaine; tentatives de structurer l'industrie de la construction, marquée par l'instabilité d'une nuée de très petites entreprises, avec la constitution d'entreprises (coopératives) dynamiques et professionnalisées de taille moyenne...

L'aspect traditionnaliste est accentué, aux yeux de plusieurs, par l'option de l'action catholique pour un mode de gestion des rapports sociaux qui donne la primauté à la société civile et à ses organisations

\footnotetext{
34 Marc-H. Choko, Jean-Pierre Collin et Annick Germain, «Le logement et les enjeux de la transformation de l'espace urbain: Montréal, 1940-1960», deuxième partie, Urban History Review/ Revue d'histoire urbaine, 15,3 (1987).

35 Sur la dynamique des courants de pensée au Québec après la guerre, voir en particulier, Michael D. Behiels, Prelude to Quebec's Quiet Revolution: Liberalism Versus Neo-Nationalism, 1945-1960 (Montréal, McGill-Queen's University, Press, 1985).

36 Bureau international du travail, Les Coopératives d' habitation, (Genève, Études et documents, no 66, 1964), 179 p.

37 Gilles Routhier, «L'ordre du monde. Capitalisme et communisme dans la doctrine de l'École Sociale Populaire, 1930-1936», Recherches sociographiques, 22,1 (1981): 7-48.
} 
— dominées de préférence par l'Église catholique - sur l'encadrement étatique. Or, comme l'a fait valoir Gérard Bergeron ${ }^{38}$, c'est oublier que la modernisation économique et politique du Québec s'est bâtie dans le sillage des expérimentations locales de ce type.

La morale chrétienne garde, bien sûr, sa prégnance sur le discours des organisations coopératives. Mais ces dernières introduisent des mécanismes collectifs innovateurs de résolution des problèmes sociaux. Or, par effet de retour, la modernisation politique du Québec (symbolisée par l'élection du Parti libéral en juin 1960) mine l'autonomie de ces organisations et de leurs modes de gestion des rapports sociaux. Les coopératives d'habitation générées par la Ligue ouvrière catholique et d'autres mouvements d'action catholique se voulaient résolument en marge de l'État, en dehors de la tutelle étatique. Pourtant, elles sont progressivement intégrées à la démarche étatique, d'autant plus aisément que les mesures qu'elles ont préconisées ne contredisaient pas cette démarche pour l'essentiel. Par contre, ces expériences tracent la voie à l'animation sociale des années soixante ${ }^{39}$.

La lecture de l'action catholique en matière d'habitation ne doit pas s'en tenir à des interprétations tranchées mais saisir les nuances et les incohérences circonstancielles, être attentive à la dualité des phénomènes. Pour y arriver pleinement, il nous faudra connaître le détail des projets, du discours, des réalisations et des échecs d'un plus grand nombre de coopératives d'habitation et savoir le rôle des militants catholiques dans ces expériences locales.

\footnotetext{
38 Gérard Bergeron et Réjean Pelletier (sous la direction de), L'État du Québec en devenir (Montréal, Boréal Express, 1980), 409 p.

39 Jean-Pierre Collin et Jacques Godbout, «Grandeur et misères des groupes populaires», Autogestions, 20-21 (1985): 53-61.
} 